\title{
Parâmetros sanguíneos e ósseos de codornas de corte alimentadas com diferentes níveis de sódio
}

\section{Blood and bone parameters of quails fed different levels of sodium}

\author{
Ednardo Rodrigues Freitas ${ }^{1 *}$; Nadja Naiara Pereira Farias ${ }^{2}$; \\ Germano Augusto Jerônimo do Nascimento ${ }^{1}$; Adriana Matos Rodrigues ${ }^{3}$; Maria \\ Goretti Rodrigues de Queiroz ; Débora Linhares Raquel²; Rafaela Castro Lima²
}

\section{Resumo}

Avaliou-se o efeito do nível de sódio da ração sobre os parâmetros sanguíneos e no desenvolvimento ósseo de codornas destinadas a produção de carne. Foram utilizadas 384 codornas italianas, distribuídas em um delineamento inteiramente casualizado, com seis tratamentos e oito repetições de oito aves por unidade experimental. Os tratamentos consistiram de rações isonutrientes, exceto para o nível de sódio, que foram: 0,$07 ; 0,12 ; 0,17 ; 0,22 ; 0,27$ e $0,32 \%$. As aves foram alimentadas de 1 a 49 dias de idade com as rações experimentais. Aos 49 dias, foram selecionadas duas aves por parcela para a coleta do sangue e retirada das tíbias. Foram avaliados o pH e a concentração de $\mathrm{Na}, \mathrm{Cl}$ e $\mathrm{K}$ no sangue e o comprimento, diâmetro, peso, índice de Seedor, teor de matéria seca e cinzas, deformidade e resistência à quebra da tíbia. Os níveis de sódio da ração não influenciaram significativamente o $\mathrm{pH}$, as concentrações de $\mathrm{Na}, \mathrm{Cl}, \mathrm{K}$ no sangue, comprimento, diâmetro, teor de matéria seca e cinzas e deformidade da tíbia. Entretanto, houve efeito quadrático sobre o peso, índice de Seedor e resistência à quebra, com ponto de máximo estimado em $0,19 \%$ de Na para todos os parâmetros. Conforme os resultados, a melhor qualidade óssea para codornas de corte foi obtida com $0,19 \%$ de sódio na ração.

Palavras-chave: Balanço eletrolítico, desenvolvimento ósseo, índice de Seedor

\begin{abstract}
We evaluated the effect of the level of sodium in the diet on blood parameters and bone development of quails for meat production. 384 Italian quails were used and distributed in a completely randomized design with six treatments and eight replicates of eight birds each. The treatments consisted of diets isonutrientes, except for the level of sodium, which were $0.07,0.12,0.17,0.22,0.27$ and $0.32 \%$. Birds were fed from 1 to 49 days old with the experimental diets and at 49 days, two birds were selected per replicate for blood collection and removal of the tibia. Were evaluated the $\mathrm{pH}$ and the concentration of $\mathrm{Na}, \mathrm{Cl}$ and $\mathrm{K}$ in the blood and the length, diameter, weight, Seedor index, dry matter content and ash content, deformity and resistance to breakage of the tibia. The sodium content of the diet did not significantly affect the $\mathrm{pH}$, concentrations of $\mathrm{Na}, \mathrm{Cl}, \mathrm{K}$ in the blood, length, diameter, dry matter and ash and deformity of the tibia. However, there was a quadratic effect on weight, Seedor index and resistance to breakage, with point of maximum estimated at $0.19 \% \mathrm{Na}$ in diet, for all parameters. As the results, the best quality bone for quails was obtained with $0.19 \%$ sodium diet.
\end{abstract}

Key words: Electrolyte balance, bone development, index Seedor

\footnotetext{
1 Profs. da Universidade Federal do Ceará, UFC, Fortaleza, CE. E-mail: ednardo@ufc.br; germanoaugusto@ufc.br; magorq@ gmail.com.br

2 Discente(s) do Programa de Pós-graduação em Zootecnia, UFC, CE. E-mail: nadja_naiara@hotmail.com; deby2502@yahoo. com.br; raffazoo@yahoo.com.br

3 Farmacêutica-Bioquímica, Laboratório de Análises Clínicas e Toxicológicas, LACT/FFOE/UFC, Fortaleza, CE. E-mail: amrodrygues@yahoo.com.br

Autor para correspondência
} 


\section{Introdução}

O sódio pertence ao grupo de minerais essenciais para as aves e exerce funções importantes no metabolismo que interferem diretamente no desempenho. Esse mineral está envolvido na regulação da pressão osmótica, no equilíbrio ácido-base, na transmissão de impulsos nervosos, no processo de absorção de nutrientes como carboidratos, aminoácidos, minerais e vitaminas e no consumo de água, tornando-o indispensável para o crescimento das aves (VIEITES et al., 2004; ARAÚJO et al., 2011; PINHEIRO et al., 2011).

A ingestão contínua de ração deficiente ou com excesso de sódio induz mudanças na concentração desse mineral nos tecidos e fluidos corporais. Em tais circunstâncias, lesões bioquímicas devem ocorrer, funções fisiológicas serão afetadas adversamente e desordens estruturais devem surgir, variando com o grau e duração da deficiência dietética ou toxidez, a idade, o sexo e a espécie animal (UNDERWOOD; SUTTLE, 1999). Segundo os pesquisadores, para prevenção dessas mudanças, o animal deve ser alimentado com dietas palatáveis e atóxicas, contendo níveis de sódio e de outros nutrientes, em quantidades e proporções exigidas e em formas disponíveis para o organismo.

O desenvolvimento adequado do sistema ósseo das aves tem sido estudado com especial atenção ao atendimento das exigências nutricionais em minerais. Entre os minerais estudados está o sódio, que juntamente com o potássio e cloro, tem grande importância no estabelecimento e manutenção da pressão osmótica e do equilíbrio ácido-básico e, por isso, podem influenciar na deposição mineral nos ossos (VIEITES et al., 2004; ARAÚJO et al., 2011). Nesse contexto, vários pesquisadores (VIEITES et al., 2004; GONZALEZ; MENDONÇA, 2006; ARAÚJO et al., 2011) têm relatado que os íons $\mathrm{Na}^{+}, \mathrm{K}^{+}$e $\mathrm{Cl}^{-}$participam da formulação das rações para as aves não só para atender os requerimentos de cada mineral, mas também para promover um balanço ótimo de eletrólitos e, assim, atender as necessidades de crescimento e evitar o aumento de problemas de pernas.

Segundo Vieites et al. (2004) e Araújo et al. (2011), os resultados das pesquisas têm demonstrado que o desequilíbrio de cátions e de ânions na ração de frangos pode ter influência na incidência de problemas de pernas havendo interação entre sódio, potássio e cloro no desenvolvimento da discondroplasia na tíbia, sendo que o elevado teor de cloro na ração tende a aumentar a incidência desse problema, sobretudo, quando os níveis de sódio e de potássio são baixos.

No Brasil é crescente o interesse pela coturnicultura de corte e, consequentemente, das pesquisas para gerar conhecimento em nutrição, que viabilizem a produção eficiente. Diante o exposto, nesse estudo objetivou-se avaliar o efeito do nível de sódio da ração sobre os parâmetros sanguíneos e no desenvolvimento ósseo de codornas destinadas à produção de carne.

\section{Material e Métodos}

Para a realização do experimento foram adquiridas 700 codornas domésticas Coturnix coturnix, da variedade italiana, de um dia de idade e de ambos os sexos com peso médio inicial de $9 \pm 0,6$ gramas. Ao chegar às instalações experimentais, as aves foram pesadas e distribuídas em seis boxes (1,0 m x 1,5 m). Em cada box, foram colocadas 101 aves que passaram a ser alimentadas com a ração correspondendo a um dos tratamentos. Todos os boxes eram providos de aquecimento com lâmpadas incandescentes de 100 watts e piso forrado com maravalha. As rações experimentais foram fornecidas em comedouros do tipo bandeja e a água em bebedouros de pássaros adaptados.

No décimo dia de idade, as aves foram novamente pesadas e, de acordo com peso médio de cada unidade experimental, 384 foram redistribuídas, em um delineamento inteiramente casualizado, com seis tratamentos, oito repetições e oito aves por unidade experimental. 
Os tratamentos consistiram de uma ração basal contendo $0,07 \%$ de sódio $(\mathrm{Na})$, suplementada com cinco níveis crescentes de sódio $(0,0 ; 0,05 ; 0,10$; $0,15 ; 0,20$ e 0,25$)$, totalizando 0,$07 ; 0,12 ; 0,17$; 0,$22 ; 0,27$ e $0,32 \%$ do elemento químico.

A ração basal (Tabela 1) foi formulada segundo as recomendações nutricionais em energia metabolizável, proteína bruta, aminoácidos, cálcio e fósforo constantes no NRC (1994). Os dados de composição dos alimentos foram baseados segundo Rostagno et al. (2005) e análises realizadas no laboratório de Nutrição Animal do Departamento de Zootecnia da Universidade Federal do Ceará.

Tabela 1. Composição nutricional das dietas experimentais.

\begin{tabular}{lrrrrrr}
\hline & \multicolumn{5}{c}{ Nível de sódio (\%) } \\
\cline { 2 - 6 } \multicolumn{1}{c}{ Ingrediente } & 0,07 & 0,12 & 0,17 & 0,22 & 0,27 & 0,32 \\
\hline Milho & 54,29 & 54,25 & 53,86 & 53,47 & 53,07 & 52,68 \\
Farelo de soja & 42,03 & 42,03 & 42,11 & 42,18 & 42,26 & 42,33 \\
Óleo de soja & 0,97 & 0,99 & 1,12 & 1,25 & 1,39 & 1,52 \\
Calcário & 1,22 & 1,22 & 1,22 & 1,22 & 1,22 & 1,22 \\
Fosfato bicálcico & 0,91 & 0,91 & 0,91 & 0,91 & 0,91 & 0,91 \\
Dl-Metionina & 0,01 & 0,01 & 0,01 & 0,01 & 0,01 & 0,01 \\
Suplemento vitamínico e mineral ${ }^{1}$ & 0,40 & 0,40 & 0,40 & 0,40 & 0,40 & 0,40 \\
Cloreto de amônia & 0,11 & 0,00 & 0,00 & 0,00 & 0,00 & 0,00 \\
Bicarbonato de sódio & 0,00 & 0,01 & 0,19 & 0,38 & 0,56 & 0,75 \\
Sal comum & 0,06 & 0,18 & 0,18 & 0,18 & 0,18 & 0,18 \\
& & & & & & \\
Composição nutricional calculada & & & & & & \\
Energia metabolizável (kcal/kg) & 2.900 & 2.900 & 2.900 & 2.900 & 2.900 & 2.900 \\
Proteína bruta (\%) & 23,80 & 23,80 & 23,80 & 23,80 & 23,80 & 23,80 \\
Lisina (\%) & 1,30 & 1,30 & 1,30 & 1,30 & 1,30 & 1,30 \\
Metionina + cistina (\%) & 0,87 & 0,87 & 0,87 & 0,87 & 0,87 & 0,87 \\
Metionina (\%) & 0,50 & 0,50 & 0,50 & 0,50 & 0,50 & 0,50 \\
Cálcio (\%) & 0,80 & 0,80 & 0,80 & 0,80 & 0,80 & 0,80 \\
Fósforo disponível (\%) & 0,30 & 0,30 & 0,30 & 0,30 & 0,30 & 0,30 \\
Sódio (\%) & 0,07 & 0,12 & 0,17 & 0,22 & 0,27 & 0,32 \\
Cloro (\%) & 0,14 & 0,14 & 0,14 & 0,14 & 0,14 & 0,14 \\
Potássio (\%) & 0,94 & 0,94 & 0,94 & 0,94 & 0,94 & 0,94 \\
NM ${ }^{2}$ (mEq/kg) & 231 & 253 & 275 & 297 & 318 & 340 \\
\hline
\end{tabular}

${ }^{1}$ Quantidade/kg do produto: ácido fólico - 138,00 mg; pantotenato de cálcio - 2.750,00 mg; antioxidante - 500,00 mg; biotina 13,80 mg; cobalto - 25,00 mg; cobre - 2.500,00 mg; colina - 111.450,00 mg; ferro - 6.250,00 mg; iodo - 260,00 mg; manganês - 13.000,00 mg; metionina - 300,00 g; niacina - 6.875,00 mg; piridoxina - 550,00 mg; colistina - 1.750,00 mg; riboflavina $1.375,00 \mathrm{mg}$; selênio - 45,00 mg; tiamina - 550,00 mg; vitamina A - 2.150.000,00 UI; vitamina B12 - 2.750,00 mcg; vitamina D3 - 550.000 UI; vitamina E - 2.750,00 UI; vitamina K - 400,00 mg; zinco - 11.100,00 mg; silicatos - 20.000,00 mg.

${ }^{2}$ Número de Mongin $(\mathrm{MONGIN}, 1981)=(\% \mathrm{Na}+\times 10000 / 22,990 *)+(\% \mathrm{~K}+\times 10000 / 39,102 *)-(\% \mathrm{Cl}-\times 10000 / 35,453 *)$ (*equivalente-grama do sódio, potássio e cloro).

Fonte: Elaboração dos autores. 
Os níveis de cloro $(\mathrm{Cl})$ e potássio $(\mathrm{K})$ foram mantidos constantes em todas as rações, sendo o nível de $\mathrm{Cl}$ mantido de acordo com as recomendações do NRC (1994) e o K oriundo dos alimentos da ração. A suplementação de sódio foi realizada com a inclusão de bicarbonato de sódio ( $\mathrm{NaHCO} 3$ ), mantendo-se o cloreto de sódio $(\mathrm{NaCl})$. Para manter os mesmos níveis de $\mathrm{Cl}$ nas rações foi utilizado o cloreto de amônia.

Durante o período de 10 a 49 dias de idade as aves foram alojadas em gaiolas de arame galvanizado $(52 \mathrm{~cm} \times 26 \mathrm{~cm} \times 20 \mathrm{~cm})$ contendo comedouros do tipo calha e bebedouros de pássaros adaptados. Por ocasião da transferência das aves do piso para as gaiolas experimentais, procedeu-se a vacinação contra a doença de Newcastle, por via ocular.

Durante o período de criação, as aves receberam 24 horas de iluminação por dia (natural e artificial), com a iluminação noturna feita por lâmpadas fluorescentes de 40 watts. Diariamente, durante todo o período experimental, a temperatura e umidade relativa do ar dentro do galpão foi medida com termômetro de máxima e mínima e psicrômetro, respectivamente. Os dados foram registrados diariamente e as leituras realizadas às $8 \mathrm{~h}$ e $16 \mathrm{~h}$. No final do experimento foram calculadas as médias das temperaturas máximas $\left(32 \pm 1,44^{\circ} \mathrm{C}\right)$ e mínimas $\left(28 \pm 1,82^{\circ} \mathrm{C}\right)$ e os valores de umidade relativa do ar de $86 \%$. As rações e a água foram fornecidas à vontade, e os comedouros e bebedouros abastecidos duas vezes ao dia, às $8 \mathrm{~h}$ e $16 \mathrm{~h}$.

Ao final do período experimental (49 dias de idade), foram selecionadas 2 aves de cada parcela experimental com peso próximo ao peso médio da parcela para a coleta de sangue e retirada das tíbias, totalizando 96 aves abatidas.

Os parâmetros sanguíneos avaliados foram: $\mathrm{pH}$ e concentração dos minerais $\mathrm{Na}(\mathrm{mmol} / \mathrm{L})$, $\mathrm{Cl}(\mathrm{mmol} / \mathrm{L}), \mathrm{K}(\mathrm{mmol} / \mathrm{L})$ e os parâmetros ósseos foram: comprimento $(\mathrm{cm})$, diâmetro $(\mathrm{cm})$ peso $(\mathrm{mg})$ índice de Seedor $(\mathrm{mg} / \mathrm{mm})$, matéria seca e cinzas $(\%)$, deformidade $(\mathrm{mm})$ e resistência $\left(\mathrm{kgf} / \mathrm{cm}^{2}\right)$.
Para a coleta de sangue, as aves das gaiolas foram retiradas e decapitadas com o auxílio de uma tesoura. O sangue de cada ave foi coletado em copo plástico descartável, sendo aproximadamente $3 \mathrm{~mL}$ colhidos com seringa e colocados imediatamente em tubos coletores de sangue contendo gel separador e deixados para coagular por cerca de 40 minutos em temperatura ambiente. Em seguida, procedeuse a centrifugação das amostras a $20000 \mathrm{rpm}$ por 15 minutos e, após a separação do soro, foram imediatamente congeladas em freezer $\left(-20^{\circ} \mathrm{C}\right)$ até as análises laboratoriais. A parte restante foi utilizada para determinação do $\mathrm{pH}$, com a imersão direta do eletrodo do pHmetro no sangue contido no copo.

As determinações de sódio, cloretos e potássio no soro sanguíneo foram realizadas no Laboratório de Análises Clínicas e Toxicológicas (LACT) da Faculdade de Farmácia, Odontologia e Enfermagem da UFC, por técnicas fotocolorimétricas utilizando kits reagentes da marca Labtest (Labtest Diagnóstica, Brasil). As determinações de sódio, potássio e cloro foram realizadas em equipamento automático (Rapidchem 744) da marca Bayer, através do princípio de íon eletrodo seletivo.

Após a sangria, foram retiradas as coxas e sobrecoxas de cada ave, identificadas, pesadas e congeladas para posterior desossa e análise dos parâmetros ósseos.

Para a realização da desossa, as peças foram descongeladas, pesadas, devidamente identificadas e mergulhadas em água fervente por 10 minutos. Em seguida foram desossadas com auxílio de um bisturi, conforme metodologia descrita por Bruno (2002).

Depois da desossa, nas tíbias do lado direito, foram medidos o comprimento e o diâmetro, com auxilio de um paquímetro digital e determinado o peso em balança de precisão $(0,01 \mathrm{~g})$. A densidade óssea $(\mathrm{mg} / \mathrm{mm})$ foi calculada através do índice de Seedor, obtido dividindo-se o valor do peso (mg) pelo comprimento $(\mathrm{mm})$ do osso avaliado (SEEDOR; QUARRACCIO; THOMPSON, 1991). 
Após a pesagem os ossos foram colocados em estufa de ventilação forçada a $55^{\circ} \mathrm{C}$ por $72 \mathrm{~h}$ para a determinação da matéria pré-seca. Em seguida, os ossos foram triturados em moinho de bola e acondicionados devidamente identificados para posterior determinação da matéria seca (MS) e matéria mineral (MM) segundo as metodologias descritas por Silva e Queiroz (2002).

A resistência e a deformidade óssea foram determinadas no osso in natura (tíbia esquerda) com auxílio de uma prensa mecânica. Os ossos foram colocados em posição horizontal sobre um suporte de madeira, apoiados sob suas extremidades, e depois foi aplicada uma força de compressão no centro de cada osso. A quantidade máxima de força aplicada no osso antes da sua ruptura foi considerada a resistência a quebra $\left(\mathrm{kgf} / \mathrm{cm}^{2}\right)$, sendo esta mensurada através de um extensômetro digital. A deformidade ( $\mathrm{mm}$ ) também foi mensurada através de um extensômetro no momento da ruptura do osso.

A análise estatística foi realizada utilizando o programa estatístico Statistical Analysis System (SAS, 2000), sendo os dados submetidos a analise de regressão, para estimativas dos efeitos dos níveis de sódio da ração sobre as variáveis estudadas.

\section{Resultados e Discussão}

Conforme a análise dos dados (Tabela 2) os níveis de sódio da ração não influenciaram significativamente o $\mathrm{pH}$ e as concentrações de $\mathrm{Na}$, $\mathrm{Cl}, \mathrm{K}$ no soro do sangue.

Tabela 2. pH e eletrólitos do sangue de codornas italianas destinadas à produção de carne alimentadas com diferentes níveis de sódio na ração.

\begin{tabular}{ccccc}
\hline Níveis de Sódio (\%) & $\mathrm{pH}$ & $\mathrm{Na}(\mathrm{mmol} / \mathrm{L})$ & $\mathrm{Cl}(\mathrm{mmol} / \mathrm{L})$ & $\mathrm{K}(\mathrm{mmol} / \mathrm{L})$ \\
\hline 0,07 & 7,12 & 140,67 & 107,61 & 3,08 \\
0,12 & 7,20 & 142,49 & 109,68 & 2,99 \\
0,17 & 7,09 & 140,67 & 106,63 & 2,90 \\
0,22 & 6,98 & 144,20 & 110,14 & 2,89 \\
0,27 & 7,10 & 140,67 & 109,29 & 2,70 \\
0,32 & 7,12 & 146,64 & 112,28 & 2,72 \\
Média & 7,10 & 142,64 & 109,45 & 2,88 \\
CV (\%) & 2,05 & 6,42 & 4,31 & 12,66 \\
Regressão & $\mathrm{NS}$ & $\mathrm{NS}$ & $\mathrm{NS}$ & $\mathrm{NS}$ \\
\hline
\end{tabular}

$\mathrm{CV}=$ Coeficiente de Variação; NS = Efeito estatístico não significativo $(\mathrm{P}>0,05)$.

Fonte: Elaboração dos autores.

A manutenção dos parâmetros sanguíneos, mesmo com o acréscimo de sódio na ração, pode ser atribuída às mudanças no comportamento das codornas, no consumo de ração e de água, em resposta às alterações nos níveis desse mineral na ração, na tentativa de manter o equilíbrio eletrolítico e a homeostase corporal, pois o consumo de ração das aves utilizadas nesse estudo aumentou com a adição de sódio na ração até o nível estimado de
$0,256 \%$ e reduziu com níveis superiores, enquanto, o consumo de água aumentou linearmente com o acréscimo de sódio na ração, conforme os dados apresentados por Raquel et al. 2011.

Segundo Mushtaq et al. (2005), naturalmente, o excesso de sódio na ração leva a respostas fisiológicas para manter a homeostase corporal. Entre elas estão os ajustes na ingestão dos eletrólitos, 
modificando o consumo de ração, o aumento da ingestão e excreção de água e redução na taxa de filtração glomerular nos rins, levando a um aumento da excreção renal de sódio.

Os resultados obtidos para codornas são semelhantes aos relatados por Mushtaq et al. (2005) para frangos de corte na fase de 1 a 28 dias de idade. Esses pesquisadores verificaram que o $\mathrm{pH}$ e as concentrações de $\mathrm{Na}$ e $\mathrm{K}$ do sangue não foram influenciados pelo nível de sódio da ração. Entretanto Mushtaq et al. (2007) constataram que o aumento de $0,20 \%$ para $0,30 \%$ de sódio na ração de frangos de corte submetidos ao estresse calórico na fase de 29 a 42 dias de idade promoveu aumento do $\mathrm{pH}$ e redução nas concentrações $\mathrm{K}$ do sangue, enquanto o $\mathrm{Na}$ não variou significativamente.

Considerando que as mudanças nos parâmetros sanguíneos das aves em resposta ao aumento dos níveis de sódio da ração podem variar em função das condições de criação, deve-se ressaltar que nem sempre é possível oferecer todas as condições para que as aves consigam manter o equilíbrio eletrolítico e, dessa forma, é necessário fornecer níveis de sódio para manter o mínimo de excreção renal desse mineral. Por sua vez, o aumento da excreção de água para eliminar o excesso de sódio pode contribuir para piorar as condições no ambiente de criação.

Conforme a análise dos dados dos parâmetros ósseos avaliados (Tabela 3) os níveis de sódio da ração não influenciaram significativamente o comprimento, diâmetro, teor de matéria seca e cinzas, bem como deformidade da tíbia. Entretanto, houve efeito quadrático sobre o peso do osso ( $\mathrm{Y}=$ $\left.492,87+2715,63 X-7008,93 X^{2}, r^{2}=0,68\right)$, índice de Seedor $\left(\mathrm{Y}=9,43+45,56 \mathrm{X}-119,43 \mathrm{X}^{2}, \mathrm{r}^{2}=\right.$ $0,63)$ e resistência à quebra $(\mathrm{Y}=0,796+10,727 \mathrm{X}$ $\left.-27,65 \mathrm{X}^{2} ; \mathrm{r}^{2}=0,72\right)$. Conforme os resultados, o peso do osso, o índice de Seedor e a resistência à quebra aumentaram à medida que o nível de sódio aumentou na ração atingindo os valores máximos para o nível de $0,19 \%$.

Tabela 3. Efeito dos diferentes níveis de sódio na ração sobre os parâmetros ósseos avaliados na tíbia de codornas italianas.

\begin{tabular}{ccccccccc}
\hline $\begin{array}{c}\text { Níveis de } \\
\text { Sódio (\%) }\end{array}$ & $\begin{array}{c}\text { Comprimento } \\
(\mathrm{cm})\end{array}$ & $\begin{array}{c}\text { Diâmetro } \\
(\mathrm{cm})\end{array}$ & $\begin{array}{c}\text { Peso } \\
(\mathrm{mg})\end{array}$ & $\begin{array}{c}\text { Índice de } \\
\text { Seedor } \\
(\mathrm{mg} / \mathrm{mm})\end{array}$ & $\begin{array}{c}\text { Matéria } \\
\text { seca } \\
(\%)\end{array}$ & $\begin{array}{c}\text { Cinzas } \\
(\%)\end{array}$ & $\begin{array}{c}\text { Deformidade } \\
(\mathrm{mm})\end{array}$ & $\begin{array}{c}\text { Resistência } \\
\left(\mathrm{kgf} / \mathrm{cm}^{2}\right)\end{array}$ \\
\hline 0,07 & 53,95 & 0,328 & 671,25 & 12,47 & 84,27 & 47,36 & 1,22 & 1,44 \\
0,12 & 54,49 & 0,303 & 692,50 & 12,69 & 84,83 & 49,33 & 1,21 & 1,46 \\
0,17 & 54,93 & 0,301 & 712,50 & 12,99 & 85,11 & 49,48 & 1,27 & 1,68 \\
0,22 & 54,76 & 0,303 & 795,00 & 14,53 & 84,69 & 49,95 & 1,36 & 1,75 \\
0,27 & 54,91 & 0,301 & 733,75 & 13,35 & 83,62 & 49,25 & 1,05 & 1,19 \\
0,32 & 54,53 & 0,318 & 623,75 & 11,41 & 84,55 & 48,85 & 1,10 & 1,11 \\
Média & 54,59 & 0,309 & 704,79 & 12,90 & 84,51 & 49,04 & 1,20 & 1,43 \\
CV (\%) & 3,78 & 9,45 & 12,88 & 12,04 & 5,97 & 5,36 & 25,84 & 34,36 \\
Regressão & $\mathrm{NS}$ & $\mathrm{NS}$ & $\mathrm{Q}$ & $\mathrm{Q}$ & $\mathrm{NS}$ & $\mathrm{NS}$ & $\mathrm{NS}$ & $\mathrm{Q}$ \\
\hline
\end{tabular}

$\mathrm{CV}=$ Coeficiente de Variação; $\mathrm{NS}=$ Efeito estatístico não significativo; $\mathrm{Q}=$ Efeito quadrático significativo $(\mathrm{P}<0,05)$.

Fonte: Elaboração dos autores. 
Segundo Underwood e Suttle (1999), a ingestão contínua de rações deficientes ou com excesso de sódio altera a concentração desse mineral nos tecidos e fluidos corporais, causando lesões bioquímicas que afetam as funções fisiológicas, conduzindo ao surgimento de desordens metabólicas relacionadas com o metabolismo proteico, energético e mineral. Nesse contexto, pode-se inferir que, embora não tenham sido verificadas mudanças significativas nos parâmetros sanguíneos, as variações no índice de Seedor e na resistência à quebra indicaram, respectivamente, prejuízos na densidade $\mathrm{e}$ resistência óssea com níveis reduzidos e elevados de sódio na ração, sendo estes resultados decorrentes de alterações fisiológicas promovidas pela baixa ou elevada ingestão desse mineral ao longo do tempo.

O osso funciona como um sistema tamponante para o controle ácido-base dos fluídos corporais estando, assim, sujeito a modificações. Na literatura (VIEITES et al., 2004; OLIVEIRA; ARANTES; STRINGHINI, 2010; ARAÚJO et al., 2011) tem sido relatado que dietas ácidas ou de baixo balanço eletrolítico, induzem a liberação de cátions do osso para o sangue para corrigir o $\mathrm{pH}$, sendo o cálcio o principal cátion liberado. Assim, a reabsorção óssea resulta em sua menor mineralização e, consequentemente, em menor peso, densidade e resistência. Isso justifica a menor qualidade óssea das codornas alimentadas com níveis menores de sódio na ração, que se tornaram mais ácidas, visto que o balanço eletrolítico das rações com 0,07 ; 0,$12 ; 0,17 ; 0,22 ; 0,27$ e $0,32 \%$ de sódio foram 231 , $253,275,297,318$ e $340 \mathrm{mEq} / \mathrm{Kg}$, respectivamente.

Araújo et al. (2011) verificaram que a redução no balanço eletrolítico da ração de frangos de corte influenciou negativamente a mineralização e resistência óssea, sendo a redução na resistência à quebra dos ossos associada não apenas às alterações na mineralização, mas também devido à mudanças na composição da matriz orgânica do osso devido à alterações nas concentrações de proteínas colagenosas e não colagenosas, que estão correlacionadas negativamente com a resistência à quebra.
Por sua vez, a melhora na qualidade óssea como acréscimo de sódio na ração se deve à redução da liberação de cátions do osso e, consequentemente, menor perda de cálcio.

Em níveis elevados, o excesso de sódio da ração induz à respostas como o aumento da ingestão de água para a excreção renal do excesso desse cátion, mantendo o equilíbrio dos eletrólitos no sangue (MUSHTAQ et al., 2005). No entanto, esse efeito pode acarretar indiretamente aumento da perda de cálcio, pois o sódio é excretado juntamente com o cálcio. Ao longo do tempo, isso vai reduzir os níveis de cálcio necessários para os ossos, levando a uma deficiência de cálcio e comprometimento da qualidade do osso.

Alterações nos níveis de cálcio no sangue de frangos de corte em resposta a modificações do balanço eletrolítico (BE) na ração foram relatadas por Vieites et al. (2004). Segundo os pesquisadores, os tratamentos com os menores e os maiores valores de BE apresentaram as maiores concentrações de fósforo e de cálcio no sangue, que podem representar maior mobilização óssea desses minerais por ação hormonal, com a finalidade de disponibilizar mais eletrólitos no sangue para serem utilizados por mecanismos compensatórios de regulação no organismo das aves.

Considerando a estimativa para o melhor nível de sódio na ração, o melhor balanço eletrolítico para obtenção de maior qualidade óssea para codornas de corte está em torno de $283 \mathrm{mEq} / \mathrm{Kg}$. Este valor se aproxima dos recomendados para frangos de corte, pois segundo Gonzalez e Mendonça (2006), o balanço eletrolítico ótimo para reduzir os problemas locomotores em frangos de corte, deve estar entre 250 e $300 \mathrm{mEq} / \mathrm{kg}$, corroborando com Johnson e Karunajeewa (1985) onde concluíram que um balanço de eletrólitos na dieta menor que $180 \mathrm{mEq} /$ $\mathrm{kg}$ e maior que $300 \mathrm{mEq} / \mathrm{kg}$ reduziu o peso das aves, quando avaliado aos 42 dias de idade, no entanto, um ótimo balanço eletrolítico foi encontrado para rações contendo de 250 a $300 \mathrm{mEq} / \mathrm{kg}$. 


\section{Conclusões}

O aumento de 0,07 para $0,32 \%$ do sódio na ração de codornas de corte não altera o $\mathrm{pH}$ e as concentrações de eletrólitos no sangue. Entretanto, a melhor qualidade óssea pode ser obtida com rações com níveis de $0,19 \%$ de sódio.

\section{Referências}

ARAÚJO, G. M.; VIEITES, F. M.; BARBOSA, A. A.; CARAMORI JUNIOR, J. G.; SANTOS, A. L.; MORAES, G. H. K.; ABREU, J. G.; MULLER, E. S. Variação aniônica da dieta sobre características ósseas de frangos de corte: resistência à quebra, composição orgânica e mineral. Arquivo Brasileiro de Medicina Veterinária e Zootecnia, Belo Horizonte, v. 63, n. 4, p. 954-961, 2011.

BRUNO, L. G. D. Desenvolvimento ósseo em frangos de corte: influência da restrição alimentar e da temperatura ambiente. 2002. Tese (Doutorado em Zootecnia, Produção Animal) - Universidade Estadual Paulista, Jaboticabal.

GONZALEZ, E.; MENDONÇA, C. X. Problemas locomotores em frangos de corte. In: SIMPÓSIO BRASIL SUL DE AVICULTURA, 7., 2006, Chapecó. Anais... Chapecó: Embrapa Suínos e Aves, 2006. p. 7994.

JOHNSON, R. J.; KARUNAJEEWA, H. The effects of dietary minerals and electrolytes on the growt and physiology of the young chick. Journal of Nutrition, Bethesda, v. 115, n. 12, p. 1680-1690, 1985.

MONGIN, P. Recent advances in dietary cation-anion balance: applications in poultry. Proceedings Nutrition Society, Cambridge, v. 40, n. 3, p.285-294, 1981.

MUSHTAQ, T.; MIRZA, M. A.; ATHAR, M.; HOOGE, D. M.; AHMAD, T.; AHMAD, G.; MUSHTAQ, M. M. H.; NOREEN, U. Dietary sodium and chloride for twentynine to forty-twoday-old broiler chickens at constant electrolyte balance under subtropical summer conditions. Journal of Applied Poultry Research, Champaign, v. 16, n. 2, p. 161-170, 2007.

MUSHTAQ, T.; SARWAR, M.; NAWAZ, H.; MIRZA, M. A.; AHMAD, T. Effect and interactions of dietary sodium and chloride on broiler starter performance (hatching to twenty-eight days of age) under subtropical summer conditions. J. Poultry Science, Honduras, v. 84, n. 11, p. 1716-1722, 2005.
NATIONAL RESEARCH COUNCIL - NRC. Nutrient requirements of poultry. 9. ed. rev. Washington: National Academy Press, 1994. 155 p.

OLIVEIRA, M. C.; ARANTES, U. M.; STRINGHINI, J. H. Efeito do balanço eletrolítico da ração sobre parâmetros ósseos e da cama de frango. Biotemas, Florianópolis, v. 23, n. 1, p. 203-209, 2010.

PINHEIRO, S. R. F.; SAKOMURA, N. K.; KAWAUCHI, I. M.; BONATO, M. A.; DORIGAM, J. C. P.; FERNANDES, J. B. K. Níveis de cloreto de sódio para aves de corte da linhagem Colonial criadas em semiconfinamento. Revista Brasileira de Zootecnia, Viçosa, MG, v. 40, n. 7, p. 1545-1553, 2011.

RAQUEL, D. L.; LIMA, R. C.; FREITAS, E. R.; NASCIMENTO, G. A. J.; SÁ, N. L.; PAIVA, A. C. Níveis de sódio para codornas italianas destinadas à produção de carne. Revista Brasileira de Zootecnia, Viçosa, MG, v. 40, n. 1, p. 1354-141, 2011.

ROSTAGNO, H. S.; ALBINO, L. F. T.; DONZELE, J. L.; GOMES, P. C.; OLIVEIRA, R. F.; LOPES, D. C.; FERREIRA, A. S.; BARRETO, S. L. T. Tabelas Brasileiras para aves e suínos: composição de alimentos e exigências nutricionais. 2. ed. Viçosa: UFV, 2005. 186 p.

SEEDOR, J. G.; QUARRACCIO, H. H.; THOMPSON, D. D. The bisphosphonate alendronate (MK-217) inhibits bone loss due to ovariectomy in rats. Journal Bone and Mineral Research, Washington, v. 6, n. 4, p. 339-346, 1991.

SILVA, D. J.; QUEIROZ, A. C. Análise de alimentos, métodos químicos e biológicos. 2. ed. Viçosa: Editora UFV, 2002. $235 \mathrm{p}$.

STATISTICALANALISYS SYSTEM - SAS. SAS/ STAT: user's guide. Version 7.0. Cary, 2000. 325 p.

UNDERWOOD, E. J.; SUTTLE, N. F. General introduction. In:__. Mineral nutrition of livestock. Wageningen: CABI, 1999. p. 1-16.

VIEITES, F. M.; MORAES, G. H. K.; ALBINO, L. F. T.; ROSTAGNO, H. S.; RODRIGUES, A. C.; SILVA, F. Á.; ATENCIO, A. Balanço eletrolítico e níveis de proteína bruta sobre parâmetros sanguíneos e ósseos de frangos de corte aos 21 dias de idade. Revista Brasileira de Zootecnia, Viçosa, MG, v. 33, n. 6, p. 1520-1530, 2004. 